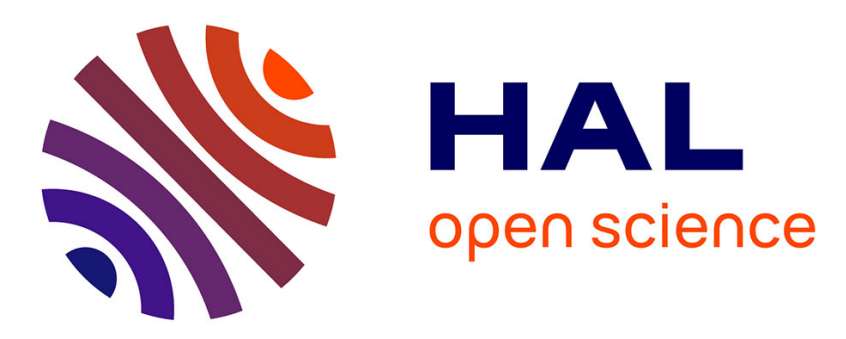

\title{
An original dynamic tribotest to discriminate friction and viscous damping
}

Emmanuel Rigaud, Joël Perret-Liaudet, Michel Belin, Lucile Joly-Pottuz, Jean-Michel Martin

\section{- To cite this version:}

Emmanuel Rigaud, Joël Perret-Liaudet, Michel Belin, Lucile Joly-Pottuz, Jean-Michel Martin. An original dynamic tribotest to discriminate friction and viscous damping. Tribology International, 2010, 43 (1-2), pp.320-329. 10.1016/j.triboint.2009.06.011 . hal-02068732

\section{HAL Id: hal-02068732 https://hal.science/hal-02068732}

Submitted on 15 Mar 2019

HAL is a multi-disciplinary open access archive for the deposit and dissemination of scientific research documents, whether they are published or not. The documents may come from teaching and research institutions in France or abroad, or from public or private research centers.
L'archive ouverte pluridisciplinaire HAL, est destinée au dépôt et à la diffusion de documents scientifiques de niveau recherche, publiés ou non, émanant des établissements d'enseignement et de recherche français ou étrangers, des laboratoires publics ou privés. 


\section{An original dynamic tribotest to discriminate friction and}

\section{viscous damping}

RIGAUD Emmanuel, PERRET-LIAUDET Joël, BELIN Michel, JOLY-POTTUZ Lucille,

$$
\text { MARTIN Jean-Michel }
$$

Laboratoire de Tribologie et Dynamique des Systèmes, UMR 5513,

Ecole Centrale de Lyon

36, avenue Guy de Collongue, 69134 Ecully Cedex, France.

Corresponding author: emmanuel.rigaud@ec-lyon.fr

Tel: +33472186296 - Fax: +33478433383 


\section{Introduction}

Controlling friction is a major issue in the context of energy consumption reductions, especially in the area of transportation. The search for tribological systems with low friction coefficients represents one of the current challenges in tribology. The friction coefficient is defined as the ratio of the contact overall friction force to the contact normal load. In practice, neither are measured directly. They are usually deduced from tangential and normal forces measured through a transducer separated from one solid involved in contact by an assembly of mechanical parts. When the shear strength of the interface is very low, measurement of the friction coefficient becomes difficult, because of the accuracy of measuring devices. The system may be perturbed by misalignment of the force transducer axes relative to the interface $[1,2]$, or by the dynamic response of the set composed by the solid involved in contact, the mechanical assembly and the transducer. Friction-induced vibrations may lead to noise measurement larger than the signal $[3,4]$.

Furthermore, for most of mechanical systems, several sources of dissipation exist simultaneously (See for example [5]). The main sources usually correspond to friction and viscous damping related to viscosity of lubricants. Nevertheless, conventional tribometers allow measurement of the overall coefficient of friction, without being able to distinguish velocity-dependent and velocity-independent contributions, from a single experiment. Results may depend on rolling and sliding contact velocities, so they may be valid only for the kinematics selected during the test.

This study deals with an original alternative way which allows to identify linear velocity-dependent and velocity-independent friction contributions, without measuring any tangential force. It is based on the analysis of the dynamic free response of a damped single degree-of-freedom oscillator, corresponding to one of the solids involved in a sliding hertzian contact.

Jacobsen and Ayre [6] proposed analytical solution for the dynamic response of a system with both viscous and Coulomb damping and developed an approximate scheme to estimate both parameters from free-vibration decrements. Liang and Feeny $[7,8,9]$ proposed an exact estimation scheme to estimate these parameters from successive peaks of the free vibration displacement or acceleration response. In this study, equivalent viscous damping $\zeta$ and kinematic friction $\mu_{\mathrm{k}}$ coefficients are identified from the retrograde integral of the energy decay curve of the dynamic system. In order to validate the methodology, an original experimental device is built, corresponding to a sphere-on-flat friction machine. Free oscillations of the spherical pin connected to a biblade are 
generated. Both velocity and displacement responses of the spherical pin are measured simultaneously from a laser velocimeter, in order to identify both kinetic and potential energies, and then energy decay curve of the dynamic system. The capability of the built device is illustrated by performing experiments with a tribological system corresponding to steel surfaces lubricated by glycerol, for several contact pressures. The objective is to show how the proposed methodology allows an accurate estimation of friction and viscous damping coefficients.

\section{Nomenclature}

a dimensionless response amplitude

$c_{0}$ viscous damping coefficient without contact

$f_{g}$ mass fraction of glycerol

$k$ spring stiffness

$m$ mass

$x$ displacement response

$y$ dimensionless displacement response

$y^{\prime}$ dimensionless velocity response

$E$ dimensionless energy decay

$N$ applied normal load

$Y_{0}$ initial dimensionless displacement

$Y_{n}$ dimensionless amplitude of peak $n$

$\beta$ dimensionless parameter depending on equivalent viscous damping

$d_{k}$ velocity-dependent part of the kinetic friction coefficient

$\varphi$ dimensionless response phase

$\mu$ overall friction coefficient

$\mu_{S}$ static friction coefficient

$\mu_{k}$ velocity-independent part of the kinetic friction coefficient

$v$ kinematic viscosity of glycerol-water solution

$v_{g}$ kinematic viscosity of glycerol

$v_{w}$ kinematic viscosity of water

$\tau$ dimensionless time 
$\tau_{0}$ initial dimensionless time

$\omega$ dimensionless circular natural frequency

$\zeta$ overall equivalent viscous damping coefficient

So equivalent viscous damping coefficient without contact

$\zeta_{k}$ equivalent viscous damping coefficient associated to velocity-dependent friction contribution

$\Delta_{\Pi}$ average energy decay curve error

$\Pi$ average energy decay

$\Omega$ circular natural frequency

\section{Theoretical background}

\subsection{Assumptions}

In this section, free motion of a single-degree-of-freedom mechanical oscillator with both friction and linear viscous underdamping is studied. The system is shown in Fig. 1. The equation of motion is:

$$
m \ddot{x}+c_{0} \dot{x}+k x=-\mu(\dot{x}) N
$$

where $x$ is the displacement of the system, $m$ is the mass, $c_{0}$ is the viscous damping coefficient without contact, $k$ is the spring stiffness, $N$ is the applied normal load and $\mu$ is the overall friction coefficient. The following form is assumed for the friction coefficient $\mu$ :

$$
\begin{array}{cc}
\mu(\dot{x})=\mu_{S} & \text { if } \dot{x}=0 \\
\mu(\dot{x})=\mu_{K} \operatorname{sgn}(\dot{x})+d_{k} \dot{x} & \text { if } \dot{x} \neq 0
\end{array}
$$

where coefficient $\mu_{\mathrm{S}}$ is associated to static friction, coefficient $\mu_{\mathrm{K}}$ is the constant part of the kinetic friction coefficient, independent of sliding velocity, and coefficient $d_{k}\left(s \cdot m^{-1}\right)$ is the linear velocitydependent part of the kinetic friction coefficient (see fig. 2).

Eq. (1) becomes:

$$
m \ddot{x}+\left(c_{0}+N \cdot d_{k}\right) \dot{x}+k x=-\mu_{K} \operatorname{sgn}(\dot{x}) \cdot N
$$

$c_{0}$ and velocity-dependent friction contribution $N . d_{k}$ can be discriminated by performing previous experiments in order to identify the free damped oscillations of the system without contact.

\subsection{Equilibrium state}

Condition for the equilibrium state can be easily found as follows: 


$$
\ddot{x}=0, \quad \dot{x}=0, \quad|x| \leq x_{S}=\frac{\mu_{S} N}{k}
$$

\subsection{Dimensionless equation of motion}

According to the Vaschy-Buckingham or $\pi$-theorem, the problem is governed by 3 dimensionless parameters, i.e. the equivalent viscous damping coefficient $\zeta$, and the two friction coefficients $\mu_{S}$ and $\mu_{K}$

Letting:

$$
\Omega^{2}=\frac{k}{m}, 2 \zeta=\frac{c_{0}+N \cdot d_{k}}{\sqrt{k m}}, \tau=\Omega t \text { and } y=\frac{k x}{N}
$$

dimensionless equation of motion is:

$$
y^{\prime \prime}+2 \zeta y^{\prime}+y=-\mu\left(y^{\prime}\right)
$$

In this equation, prime represents derivative respect to the dimensionless time $\tau$. In dimensionless form, equilibrium state becomes:

$$
y^{\prime \prime}=0, \quad y^{\prime}=0, \quad|y| \leq \mu_{S}
$$

The equivalent viscous damping coefficient can be decomposed as follows:

$$
\zeta=\zeta_{0}+\zeta_{k}
$$

with

$$
2 \zeta_{0}=\frac{c_{0}}{\sqrt{k m}}
$$

and

$$
2 \zeta_{k}=\frac{N \cdot d_{k}}{\sqrt{k m}}
$$

where $\zeta_{0}$ is the equivalent viscous damping without contact and $\zeta_{k}$ is an equivalent viscous damping coefficient corresponding to the velocity-dependent friction contribution of the tribological system.

\subsection{Free vibrations}

The exact solution of Eq. (7) can be built piecewisely, according to the sign of $y^{\prime}$. With the underdamping assumption, i.e. $0<\zeta<1$, displacement response is:

$$
y+\mu_{K} \operatorname{sgn}\left(y^{\prime}\right)=\left[Y_{0}+\mu_{K} \operatorname{sgn}\left(y^{\prime}\right)\right] e^{-\zeta\left(\tau-\tau_{0}\right)}\left[\cos \left(\omega\left(\tau-\tau_{0}\right)\right)+\beta \sin \left(\omega\left(\tau-\tau_{0}\right)\right)\right]
$$

with

$$
\omega=\sqrt{1-\zeta^{2}}
$$




$$
\beta=\frac{\zeta}{\sqrt{1-\zeta^{2}}}
$$

And

$$
Y_{0}=y\left(\tau_{0}\right), \quad y^{\prime}\left(\tau_{0}\right)=0
$$

The solution is valid until $y^{\prime}=0$ which occurs at time $\tau_{1}=\tau_{0}+\pi / \omega$. If $Y_{0}>0$ (respectively $Y_{0}<0$ ), motion starts with $y^{\prime}<0$ (respectively $y^{\prime}>0$ ). Solution can be expanded for all periods between $\tau_{n}$ and $\tau_{n+1}=\tau_{n}+\pi / \omega$, such as $y^{\prime}\left(\tau_{n}\right)=0$, as follows:

$$
y(\tau)=\mu_{k} \operatorname{sgn}\left(Y_{n}\right)+\left(Y_{n}-\mu_{k} \operatorname{sgn}\left(Y_{n}\right)\right) e^{-\zeta\left(\tau-\tau_{n}\right)}\left[\cos \left(\omega\left(\tau-\tau_{n}\right)\right)+\beta \sin \left(\omega\left(\tau-\tau_{n}\right)\right)\right]
$$

and

$$
y^{\prime}(\tau)=-\frac{Y_{n}-\mu_{k} \operatorname{sgn}\left(Y_{n}\right)}{\omega} e^{-\zeta\left(\tau-\tau_{k}\right)} \sin \left(\omega\left(\tau-\tau_{n}\right)\right)
$$

with

$$
Y_{n}=y\left(\tau_{n}\right)
$$

and

$$
\operatorname{sgn}\left(Y_{n}\right)=-\operatorname{sgn}\left(y^{\prime}\right)
$$

Consequently, the phase planes are obtained. Trajectories consist of a series of elliptical arcs centred alternatively at point $\left(y=-\mu_{k}, y^{\prime}=0\right)$ and point $\left(y=+\mu_{k}, y^{\prime}=0\right)$. The motion ceases as soon as the trajectory intersects the $y$-axis between the two points defined by $y= \pm \mu_{s}$. Fig. 3 shows an example of trajectory in the phase plane, whereas the corresponding time histories of displacement and velocity are given in Fig. 4.

\subsection{Decaying law of vibration}

Damping parameters can be deduced from the decaying law of the successive peaks of the free response. For example, system subjected to purely and constant viscous damping provides peaks with exponential decay. The equivalent viscous damping coefficient is estimated from logarithmic decrement of peaks [10]. Similarly, system subjected to purely and constant Coulomb friction provides peaks with linear decay and friction coefficient is estimated from linear decrement of peaks [11]. From Eq. (16) and after some simple arrangements, the relation between successive negative and positive peaks of a system subjected to both friction and viscous damping is obtained as follows:

$$
Y_{n+1}=-Y_{n} \exp (-\beta \pi)+\mu_{k} \operatorname{sgn}\left(Y_{n}\right)(1+\exp (-\beta \pi))
$$

Feeny and Liang $[7,8]$ proposed to identify the two parameters $\beta$ and $\mu_{\mathrm{k}}$ on the basis of this decaying law Eq. (20). $\beta$ is estimated in a first step, by examining the evolution of the sum of successive peak values, leading to the following expression: 


$$
\beta=-\frac{1}{2 p \pi} \operatorname{Ln}\left(\frac{-Y_{2 p}-Y_{2 p+1}}{Y_{0}+Y_{1}}\right)
$$

where $p$ is an integer. The equivalent viscous damping coefficient $\zeta$ is then deduced from $\beta$ and Eq. (14). The kinematic friction coefficient $\mu_{\kappa}$ is deduced in a second step, from Eq. (20). Method has been recently extended to vibration acceleration decrements [9].

1.6 Identification of the damping parameters from non successive peaks

If dissipation forces are very low, the two maxima $Y_{p}$ and $Y_{p+1}$ of one oscillation are very close, because signal decreasing is very slow. So, results are very sensitive to measurement errors and experimental noise. The lower the damping is, the larger the error obtained. One way to improve the above technique is to consider the dynamic response of the system over several periods, and non successive peaks $Y_{0}, Y_{2 p}$ and $Y_{2 q}$ of the displacement response (or peaks of the velocity response, using $-Y^{\prime}{ }_{k}$ in place of $Y_{k}$.

Following equations are deduced from Eq. (20):

and

$$
\begin{aligned}
& Y_{2}=Y_{0} \exp (-2 \pi \beta)-\mu_{\kappa} \exp (-2 \pi \beta)-2 \mu_{\kappa} \exp (-\pi \beta)-\mu_{\kappa} \\
& Y_{2 p}=Y_{0} \exp (-2 \pi p \beta)-\mu_{k}\left(1+\exp (-2 \pi \beta)+2 \sum_{i=1}^{2 p-1} \exp (-i \pi \beta)\right) \\
& \mu_{k}=\frac{Y_{0} \exp (-2 \pi p \beta)-Y_{2 p}}{1+\exp (-2 \pi \beta)+2 \sum_{i=1}^{2 p-1} \exp (-i \pi \beta)}
\end{aligned}
$$

$\beta$ can be calculated from solving the following equation (where $p$ and $q$ are distinct integers):

$$
\frac{Y_{0} \exp (-2 \pi p \beta)-Y_{2 p}}{1+\exp (-2 \pi \beta)+2 \sum_{i=1}^{2 p-1} \exp (-i \pi \beta)}=\frac{Y_{0} \exp (-2 \pi q \beta)-Y_{2 q}}{1+\exp (-2 \pi \beta)+2 \sum_{i=1}^{2 q-1} \exp (-i \pi \beta)}
$$

$\zeta$ is then deduced from $\beta$, and $\mu_{\mathrm{k}}$ is deduced from Eq. (24). For low dissipation forces, dynamic response presents a large number of oscillations before extinction, so that non successive peaks may be selected such that they have very different amplitudes.

1.7 Energy decay curve 
For identifying damping parameters, another more elegant way is to consider the energy decay curve. Both kinetic and potential energies of the system can be identified from simultaneous measurements of velocity and displacement responses.

$$
E(\tau)=\frac{1}{2}\left(y^{2}+y^{\prime 2}\right)
$$

The energy decay curve is monotonic and contains all the knowledge on damping properties. Then, damping parameters are no more estimated from the $\mathrm{N}$ few discrete points corresponding to the peaks used in the above approach, but from the number N' of acquisition samples and the signal duration before extinction ( $\left.\mathrm{N}^{\prime}>>\mathrm{N}\right)$.

For viscous underdamping and low kinematic friction coefficient corresponding to the studied systems, the energy decay curve of the system can be obtained with a good accuracy by using the well known averaging method (see for example [12]). In this method, the displacement and velocity responses are assumed to have the following dimensionless form:

$$
\left\{\begin{array}{l}
y(\tau)=a(\tau) \cos (\tau+\varphi(\tau))=a \cos (\phi) \\
y^{\prime}(\tau)=-a(\tau) \sin (\tau+\varphi(\tau))=-a \sin (\phi)
\end{array}\right.
$$

where amplitude $a(\tau)$ and phase $\varphi(\tau)$ vary slowly in time without affecting the oscillatory behaviour of the solution. Then, the energy decay curve $E(\tau)$ is directly related to the amplitude function:

$$
E(\tau)=\frac{a(\tau)^{2}}{2}
$$

By averaging out the variations in $\phi$, we obtain the following classical equations describing the slow variations of $a(\tau)$ and $\varphi(\tau)$ :

$$
\begin{gathered}
a^{\prime}=-\frac{1}{2 \pi} \int_{0}^{2 \pi} \sin \phi g(a \cos \phi,-a \sin \phi) \mathrm{d} \phi \\
\varphi^{\prime}=-\frac{1}{2 \pi a} \int_{0}^{2 \pi} \cos \phi g(a \cos \phi,-a \sin \phi) \mathrm{d} \phi
\end{gathered}
$$

In these equation, the function $g\left(y, y^{\prime}\right)$ represents the nonlinear function of $y$ and $y^{\prime}$. Hence

$$
g\left(y, y^{\prime}\right)=-2 \zeta y^{\prime}-\mu_{k} \operatorname{sgn}\left(y^{\prime}\right)
$$

and Eq. (29) and (30) become:

$$
a^{\prime}=-\zeta a-\frac{2 \mu_{k}}{\pi}
$$


Solving these equations yields:

$$
a(\tau)=\left(a_{0}+\frac{2 \mu_{k}}{\pi \zeta}\right) \exp (-\zeta \tau)-\frac{2 \mu_{k}}{\pi \zeta}, \quad \varphi=\varphi_{0}
$$

With the initial conditions $a_{0}=Y_{0}$.

Finally, to this level of approximation, the averaged energy decay law is:

$$
E(\tau)=\frac{1}{2}(\Lambda \exp (-\zeta \tau)-\Gamma)^{2}
$$

where

$$
\Gamma=\frac{2 \mu_{k}}{\pi \zeta}, \quad \Lambda=Y_{0}+\Gamma
$$

Remarks that Eq. (35) remains valid until the motion ceases. It is then convenient to give the associated final time $T_{f}$ which can be obtained from the following condition:

$$
\frac{d E}{d \tau}\left(T_{f}\right)=0
$$

leading to:

$$
T_{f}=-\frac{1}{\zeta} \operatorname{Ln} \frac{\Gamma}{\Lambda}
$$

Fig. 5a displays the approximate curve given by Eq. (35) compared to the exact energy decay curve obtained from the piecewise analytical solutions, Eq. (16) and (17). The slight difference is due to the residual oscillatory behaviour of the exact energy decay curve. In order to smooth this fluctuation, we introduce the retrograde integration of the energy response at time $\tau$, as proposed in [13] for the integrated impulse method to measure reverberation time in room acoustics. Then, this averaged energy decay curve $\Pi(\tau)$ can be written as follows:

$$
\Pi(\tau)=\int_{\tau}^{T_{f}} E(\theta) \mathrm{d} \theta
$$

From Eq. (35), the approximate averaged energy decay curve $\Pi_{\text {app }}(\tau)$ is easily obtained as:

$$
\Pi_{a p p}(\tau)=\frac{\Lambda^{2}}{4 \zeta} \exp (-2 \zeta \tau)-\frac{\Lambda \Gamma}{\zeta} \exp (-\zeta \tau)-\frac{\Gamma^{2}}{2} \tau+A
$$

with the constant 


$$
A=\frac{\Gamma^{2}}{\zeta}\left(\frac{3}{4}-\operatorname{Ln}\left(\frac{\Gamma}{\Lambda}\right)\right)
$$

Fig. $5 \mathrm{~b}$ shows that the exact averaged energy decay and the approximate one are very similar. This last one can be used for identifying the damping parameters $\zeta$ and $\mu_{k}$.

\section{The original dynamic tribometer}

On the basis of the theoretical background reported in the above section, an original dynamic tribometer has been designed and built, in order to discriminated the different friction contributions. It corresponds to a sphere-on-flat friction machine which allows a sliding contact between a spherical pin and a plane. The spherical pin is connected to a $35 \mathrm{~g}$ aluminium support mounted on a biblade connected to the rigid flat. The mechanical device principle is depicted Fig. 6.

\subsection{Oscillating system}

The biblade is made of $(76 \times 10 \times 0.1 \mathrm{~mm})$ steel blades. It allows oscillations of the spherical pin according to the horizontal direction. With additional mass corresponding to the spherical pin support, its first natural frequency is equal to $32.6 \mathrm{~Hz}$. The corresponding horizontal vibrations are associated to the first flexural mode eigenshape. The dimensions of two steel blades were selected in order to uncouple this mode and the first mode corresponding to vertical vibrations and governed by the Hertzian contact law [14, 15].

Materials used and connections between mechanical parts were designed in order to minimize equivalent viscous damping coefficient of the system without contact $\zeta_{0}$.

An electromagnet is used to obtain a displacement of the spherical pin far from its equilibrium position. The amplitude of the initial displacement can vary up to $2 \mathrm{~mm}$. Then, the electromagnetic force is removed in order to initiate the free response of the oscillator. The first oscillation is not taken into account in order to remove perturbation due to the magnetic force. The maximum velocity of the spherical pin can vary up to $0.4 \mathrm{~m} \cdot \mathrm{s}^{-1}$.

\subsection{Load application}

Load is applied from micrometric vertical displacement of the spherical pin support relative to the flat. Load is accommodated through elastic deformation of a steel blade corresponding to a cantileverbeam. The system has been calibrated using a precision balance in place of the flat. Evolution of measured force is linear with vertical displacement and corresponds to a variation equal to 
$0.32 \mathrm{~N} / \mathrm{mm}$. Vertical displacement range $(0-2 \mathrm{~mm})$ allows variation of the applied load from 0 to

$0.64 \mathrm{~N}$. The device has been designed such that the spherical pin motion is parallel to the flat, so that the applied load remains constant during the oscillations.

\subsection{Measuring device}

The dynamic response is identified using a laser vibrometer system which operates on the Doppler principle, measuring back-scattered laser light from the vibrating structure, to determine its vibrational velocity and displacement. It allows the simultaneous measurement of the velocity and the displacement, so that, the exact energy of the system can be acquired directly. The second advantage is that there is no extra mass adding on the experimental device. For the selected measurement ranges $(1 \mathrm{~m} / \mathrm{s}$ for the peak to peak velocity and $10 \mathrm{~mm}$ for the peak to peak displacement), the velocity resolution is $0.015 \mu \mathrm{m} / \mathrm{s}$ and the displacement resolution is $0.15 \mu \mathrm{m}$.

\subsection{Tribological system}

Experiments reported in this study have been performed with a spherical pin radius equal to $3 \mathrm{~mm}$. The opposing surfaces used are made of AISI 52100 steel. They are polished with diamond solutions $(6,3$ and $1 \mu \mathrm{m})$ to a surface roughness $R_{a}$ less than $15 \mathrm{~nm}$. From Hertzian theory, the applied load corresponds to a contact pressure up to $0.56 \mathrm{GPa}$. Experiments are performed at ambient temperature $\left(20^{\circ} \mathrm{C}\right)$.

Contact is lubricated by glycerol $\left(\mathrm{C}_{3} \mathrm{H}_{8} \mathrm{O}_{3}\right)$. This lubricant has also been chosen because it is very easy to vary and to control the kinematic viscosity over a large range by adding a mass fraction of water in the lubricant, in order to analyze its influence on viscous damping and friction coefficients. In fact, Sabot and al. [16] have observed that dissipation induced by fluid pumping phenomenon observed in an Hertzian contact excited by normal load is related to the viscosity of the fluid surrounding the contact. Glycerol has also been chosen because it leads to low friction in lubrication of steel surfaces [17].

\subsection{Dynamic tests without contact}

Experiments without contact between the spherical pin and the flat have been performed, in order to identify dynamic and damping characteristics of the test apparatus alone and the test apparatus with a lubricant meniscus. Apparatus dissipation can have several origins: inner damping inherent to the materials used, damping caused by micro-sliding in the connections between mechanical parts, 
damping caused by the acoustic radiation of vibrating surfaces and vibrations transmitted to the flat.

Fig. 7 displays oscillations of the spherical pin in the air. The modelling of the device by a single degree-of-freedom linear oscillator is validated. The vibrational behaviour is linear as dynamic response remains small relative to the biblade length, and the measured natural frequency $(32,6 \mathrm{~Hz})$ does not vary with amplitude of the dynamic response.

Fig. 7 shows that dynamic response is characterized by peaks with exponential decay, so that power dissipation corresponds to purely velocity-dependent dissipation and can be modelled by an equivalent viscous damping $\zeta_{0}$, identified from the least mean square method. The average energy decay curve error $\Delta_{\Pi}$ is defined from the experimental data and the simulated exact dynamic curve given by Eq. (26):

$$
\Delta_{\Pi}=\sqrt{\frac{1}{N} \sum_{i=1}^{N}(\Pi(\exp )-\Pi(n u m))^{2}}
$$

The result corresponding to minimization of $\Delta_{\Pi}$ is:

$$
\zeta_{0}=0.0033
$$

\section{Measurement of friction and equivalent viscous damping coefficients}

\subsection{Contact lubricated by glycerol}

We performed experiments for five different loads, from $0.07 \mathrm{~N}$ to $0.60 \mathrm{~N}$, corresponding to contact pressure from 0.28 to $0.56 \mathrm{GPa}$. The natural frequency of the tribological system is identical to that measured without contact. Once the load applied, each experiment was repeated three times. Fig. 8 displays the experimental free responses for several applied loads.

The dynamic response shows a decay that is neither linear nor exponential. Unlike the system without contact characterized by a purely viscous damping, velocity-dependent and velocity-independent friction contributions coexist in the contact.

$\zeta$ and $\mu_{\mathrm{k}}$ have been identified from the least mean square method. $\zeta, \mu_{\mathrm{k}}$ and dimensionless initial displacement values are optimized with an absolute error equal to $10^{-5}$ in order to minimize energy decay curve error $\Delta_{\Pi \text {. }}$. Fig. 9 shows that identified coefficients $\zeta$ and $\mu_{\mathrm{\kappa}}$ are almost constant with the applied load. The several experiments give the following results:

$$
\begin{aligned}
& \zeta=0.0236 \\
& \mu_{\kappa}=0.0294
\end{aligned}
$$


with standard deviations:

$$
\begin{gathered}
\sigma(\zeta)=0.0012 \\
\sigma\left(\mu_{\kappa}\right)=0.0036
\end{gathered}
$$

The equivalent viscous damping coefficient has significantly increased compared with that measured without contact $\zeta_{0}$. So, $\zeta_{k}=\zeta-\zeta_{0}$ corresponds to the linear velocity-dependent part of the contact friction coefficient, associated to shear forces in the interfacial gap.

Unlike the system without contact characterized by a purely equivalent viscous damping, the contact friction exhibits a velocity-independent friction contribution $\mu_{\mathrm{\kappa}}$, even for full-film elasto-hydrodynamic lubrication. The behavior of the lubricant under pressure is not only governed by its viscosity. It behaves partly like a solid subjected to elastic deformations. Nevertheless, friction contribution $\mu_{\mathrm{\kappa}}$ is almost constant, despite a slight variation of the contact pressure. Alternatively, velocity-independent friction may be due to non-linear velocity distribution in the interfacial gap or to slippage at the wall. Additional experiments allowing visualization of the interface are necessary to validate these assumptions.

The dissipation force induced by $\mu_{\mathrm{\kappa}}$ increases with the applied load and the signal extinction is getting faster, as confirmed by Fig. 8. Comparison between experimental results and simulations confirm that dynamic responses are very close for the whole studied loads. Displacement or velocity successive peaks are very well adjusted for the entire duration of the signal. In addition, the estimated duration of the signal before its extinction (eq. 38) is very consistent with experimental one.

Each period of the free oscillations response corresponds to different amplitude and velocity ranges, due to the decreasing of the signal. So that, evolution of frictions characteristics with amplitude range can be analyzed by moving an observation window with fixed length along the time axis [18]. These different estimations lead to pairs of values $\left(\zeta_{\kappa}, \mu_{\kappa}\right)$ substantially identical in all cases. This result confirms assumptions of a constant velocity-independent friction contribution and of a linear velocitydependent one. The entire duration of the signal before extinction can be retained for the friction coefficients estimation.

Prior experiments have been performed using a reciprocating sphere-on-flat tribometer equipped with triboscopy and data processing [19]. This tribometer allows several operating conditions, corresponding to large amplitude of motion, stationary sliding velocity and normal load ranges. The tangential force is measured with a piezoelectric transducer. The spherical pin was loaded on the 
stationary flat. The stroke length was fixed to $2.5 \mathrm{~mm}$. The tribological system has also been lubricated with glycerol $\left(\mathrm{C}_{3} \mathrm{H}_{8} \mathrm{O}_{3}\right)$. The sliding speed was very low $(0.001 \mathrm{~m} / \mathrm{s})$. At ambient temperature $\left(20^{\circ} \mathrm{C}\right)$ and for a contact pressure equal to $0.42 \mathrm{GPa}$, the overall friction coefficient measured was equal to $0.035[17]$

Estimation of tangential forces induced by both velocity-independent and velocity-dependent friction contributions shows that, for a very low sliding speed, the velocity dependant friction contribution $\zeta$ is negligible. So that the overall friction coefficient measured during these prior experiments should be compared with the velocity-independent friction contribution $\mu_{\kappa}$. Measured values are in good agreement, even if the operating conditions are different. In fact, contact pressures are almost the same, but kinematics corresponding to the two experimental systems are very different.

\subsection{Contact lubricated by glycerol-water solutions}

According to [20], viscosity of a glycerol-water solution can be represented by empirical law:

$$
\frac{\ln v-\ln v_{w}}{\ln v_{g}-\ln v_{w}}=x_{g}\left[1+\left(1-f_{g}\right)\left(a+b f_{g}+c f_{g}{ }^{2}\right)\right]
$$

where $v_{\mathrm{g}}, v_{\mathrm{w}}$ and $v$ are the kinematic viscosities of glycerol, water and glycerol-water solution and $\mathrm{f}_{\mathrm{g}}$ is the mass fraction of glycerol. At ambient temperature $\left(20^{\circ} \mathrm{C}\right), v_{\mathrm{g}}=1160 \mathrm{~mm}^{2} / \mathrm{s}, v_{\mathrm{w}}=1.01 \mathrm{~mm}^{2} / \mathrm{s}, \mathrm{a}=-$ $0.76728, b=0.12153$ and $c=-1.41519$. Five different mass fractions of water have been added in the lubricant in order to vary the kinematic viscosity from $1160 \mathrm{~mm}^{2} / \mathrm{s}$ to $200 \mathrm{~mm}^{2} / \mathrm{s}$. For each tested solution, this one has been measured using a capillary viscometer, from the flow time required for a fixed volume of the solution to drain through a capillary under gravity (see table 1). The measured kinematic viscosity are quite close to the empirical value obtained from eq. 43 [20]. As glycerol is a hygroscopic substance, the viscosity measured for initial glycerol $\left(1040 \mathrm{~mm}^{2} / \mathrm{s}\right)$ has also been used to control its purity: the estimated mass fraction of water is $0.55 \%$.

We performed experiments for each tested solution and $\zeta$ and $\mu_{\mathrm{k}}$ have been identified. Fig 10a displays the evolution of the equivalent viscous damping coefficient $\zeta$ versus kinematic viscosity. Adding a mass fraction of water in the lubricant leads to a decreasing of the equivalent viscous damping coefficient. This result confirms that the velocity-dependent part of the contact friction is related to the kinematic viscosity of the lubricant.

Fig. $10 \mathrm{~b}$ displays the evolution of the friction coefficient $\mu_{\mathrm{k}}$ versus kinematic viscosity. For a very few mass fraction of water (less than $6 \%$, corresponding to a kinematic viscosity equal to $360 \mathrm{~mm}^{2} / \mathrm{s}$ ), the 
velocity-independent friction contribution is almost the same. Therefore, signal duration before extinction is longer, compared to contact lubricated by pure glycerol, because of the decreasing of the velocity-dependent part of the contact friction.

Adding a larger mass fraction of water leads to a drastic increase of the friction coefficient $\mu_{\kappa}$, so that the dynamic response decay is more pronounced and the signal extinction is getting faster, as confirmed by Fig. 11. Friction coefficient $\mu_{\mathrm{k}}$ presents larger variations with load than for a null or a very few mass fraction of water. These phenomena are probably attributed to a transition from elastohydrodynamic full-film regime to mixed or boundary lubrication and to the occurrence of metal/metal contact. Further works including experimental devices allowing to visualize the interface and to identify film thickness and lubrication regime are necessary to confirm this last point.

\section{Conclusion}

We proposed an original methodology that allows discrimination of velocity-dependent and velocityindependent energy dissipations, from the analysis of the free response of a single degree-of-freedom oscillator. Identification of equivalent viscous damping $\zeta$ and friction $\mu_{\mathrm{k}}$ values is based on minimization of the error defined from the least squares method applied to the retrograde integrals of the energy decay curve.

An experimental device was built, in order to implement the methodology. A spherical pin on flat contact lubricated by glycerol was studied. Experiments have confirmed that, unlike the system without contact characterized by a purely viscous damping, the contact friction is characterized by both velocity-dependent and velocity-independent contributions. Despite the extent of the applied loads, we were able to get an accurate estimation of friction coefficients. Simulations of the dynamic behaviour from identified values whenever give very satisfactory results. The successive peaks are very well fitted for the entire duration of the signal. In addition, the estimated duration of the signal before its extinction is very consistent with experimental ones. Complementary experiments using glycerol-water solutions have confirmed that the velocity-dependent part of the contact friction is related to the kinematic viscosity of the lubricant.

The proposed innovative methodology appears well suited, especially for low and ultra-low friction systems. In fact, for most tribometers, measurement of the friction coefficient becomes difficult, because of the accuracy of mechanical devices measuring friction tangential force. On the contrary, the proposed methodology is very powerful because the signal duration before extinction becomes 
longer when the dissipation forces decrease. Consequently, lower the friction is, higher the accuracy of the friction coefficients evaluation.

Coupling of such measurements with electrical contact resistance measurements are currently under investigations, in order to extract information on the film thickness and the associated lubrication regime.

\section{7- Acknowledgements}

The authors would like to thanks D. Mazuyer for helpful comments and discussions and the French Research Agency for its financial support (project ANR-06-BLAN-0115).

\section{8- References}

[1] Schmitz T., Action J., Ziegert J., Sawyer G., The difficulty of measuring low friction: uncertainty analysis for friction coefficient measurements. Journal of tribology, 127, $\mathrm{N}^{\circ} 4$, pp. 673-678, 2005.

[2], Burris D., Sawyer G., Adressing practical challenges of low friction coefficient measurements. Tribology Letters, 35, N¹, pp. 17-23, 2009.

[3] Martin J.M., Donnet C., Le Mogne T., Epicier T., Superlubricity of molybdenum disulphide. Physical Review B, 48, №14, 1993.

[4] Streator J., Bogy D., Accounting for transducer dynamics in the measurement of friction. Journal of tribology, 114, N¹, pp. 86-94, 1992.

[5] Nashif A., Jones D., Anderson J., Vibration damping, John Wiley and Sons, 1985.

[6] Jacobsen L., Ayre R., Engineering Vibrations, McGraw-Hill, New York 1958.

[7] Feeny B., Liang J., A decrement method for the simultaneous estimation of Coulomb and internal friction, Journal of Sound and Vibration, 195, pp. 149-154. 1996.

[8] Liang J., Feeny B., Identifying Coulomb and viscous friction from free-vibration decrements, Nonlinear Dynamics, 16, Number 4, 337-347, 1998.

[9] Liang J., Identifying Coulomb and viscous damping from free-vibration acceleration decrements, Journal of Sound and Vibration, 282, Issues 3-5, pp. 1208-1220, 2005.

[10] Rayleigh J. The theory of sound, Vol. 1. Reprinted by Dover, New York, 1945, pp. 35-40. 1877 [11] Lorenz H., Lehrbuch der technischen physik. Technische mechanik starrer gebilde, erster band. Verlag von Julius Springer, Berlin, 1924.

[12] Nayfeh A., Mook D., Nonlinear oscillations, Wiley-Interscience, New York (1979). 
[13] Schroeder M., New method of measuring reverberation time, J. Acoust. Soc. Am., 37, pp. 409412, 1965.

[14] Rigaud, E., Perret-Liaudet, J., Experiments and numerical results on nonlinear vibrations of an impacting hertzian contact. Part 1: harmonic excitation. Journal of Sound and Vibration, $265, \mathrm{~N}^{\circ} 2$, pp. 289-307, 2003.

[15] El Kilali, T., Perret-Liaudet, J., Mazuyer, D., Experimental analysis of a high pressure lubricated contact under dynamic normal excitation force. Transient Processes in Tribology: Proceedings of the 30th Leeds-Lyon Symposium on Tribology, Lyon,Elsevier, pp. 409-418, 2004.

[16] Sabot, J., Krempf, P., Janolin, C., Non linear vibrations of a sphere-plan contact excited by a normal load. Journal of Sound and Vibration. 214, №2, pp. 359-375, 1998.

[17] Joly-Pottuz L., Martin J.M., De Barros Bouchet M.I., Belin M., Anomalous low friction under boundary lubrication of steel surfaces by polyols, Tribology Letters, 2008.

Wear, 156, pp. 151-161. 1992.

[18] Wu, Z., Liu, H., Liu, L., Yuan, D., Identification of nonlinear damping and Coulomb friction from the free response data. Journal of Sound and Vibration, 304, pp. 407-414, 2007.

[19] Belin M., Martin J.M., Triboscopy: a new approach to surface degradation of thin film. Wear, 156, pp. 151-161. 1992.

[20] Shankar P., Kumar M., Experimental determination of the kinematic viscosity of glycerol-water mixtures. Proceedings of the Royal Society: Mathematical and Physical Sciences, 444, Nº1922, pp. 573-581, 1994. 


\section{Tables and figures}

Figure 1: The viscous and friction damped mass spring oscillator.

Figure 2: Linear velocity-dependent friction coefficient.

Figure 3: Trajectory on the phase plane. $\zeta=0.05, \mu_{\mathrm{k}}=0.02, \mu_{\mathrm{s}}=0.03$ and initial conditions $\mathrm{y}=15$, $y^{\prime}=0$.

Figure 4: Displacement (a) and velocity (b) time responses. $\zeta=0.05, \mu_{\mathrm{k}}=0.02, \mu_{\mathrm{s}}=0.03$ and initial conditions $y=15, y^{\prime}=0$.

Figure 5: Energy decay curve $E(\tau)(a)$ and averaged energy decay curve $\Pi(\tau)(b)$. Exact curves $(\cdot)$ and approximate ones $(-) . \zeta=0.05, \mu_{\mathrm{k}}=0.02, \mu_{\mathrm{s}}=0.03$ and initial conditions $\mathrm{y}=15, \mathrm{y}^{\prime}=0$.

Figure 6: Schematic of the mechanical device.

Figure 7: Free response in the air without contact. Displacement response (a), velocity response (b), experimental $(\cdot)$ and simulated $(-)$ energy decay curves $(c)$, experimental $(\cdot)$ and simulated $(-)$ averaged energy decay curves (d).

Figure 8: Experimental (-) and simulated (-) velocity responses (a) and averaged energy decay curves (b). $\mathrm{N}=0.07 \mathrm{~N}, \mathrm{~N}=0.12 \mathrm{~N}, \mathrm{~N}=0.31 \mathrm{~N}, \mathrm{~N}=0.46 \mathrm{~N}$ and $\mathrm{N}=0.59 \mathrm{~N}$.

Figure 9: Evolution of the equivalent viscous damping (a) and friction (b) coefficients versus normal load.

Figure 10: Evolution of the equivalent viscous damping $\zeta$ (a) and friction $\mu_{\mathrm{k} .}$ (b) coefficients versus kinematic viscosity.

Figure 11: Experimental velocity response for $\mathrm{N}=0.46 \mathrm{~N}$. Mass fraction of water is $6 \%$ (a) and $9 \%$ (b). 


\begin{tabular}{|c|cccccc|}
\hline Mass fraction of water & $\mathbf{0 . 5 5} \%$ & $\mathbf{2 . 3} \%$ & $\mathbf{3} \%$ & $\mathbf{6} \%$ & $\mathbf{8} \%$ & $\mathbf{9 \%}$ \\
\hline Experimental kinematic viscosity $\left(\mathrm{mm}^{2} / \mathrm{s}\right)$ & 1029 & 696.4 & 600.0 & 353.3 & 257.6 & 210.7 \\
Empirical value & 1029 & 718.8 & 625.5 & 356.7 & 252.5 & 214.2 \\
Relative error & - & $3.2 \%$ & $4.1 \%$ & $1.0 \%$ & $2.0 \%$ & $1.6 \%$ \\
\hline
\end{tabular}


Fig.1: The viscous and friction damped mass spring oscillator

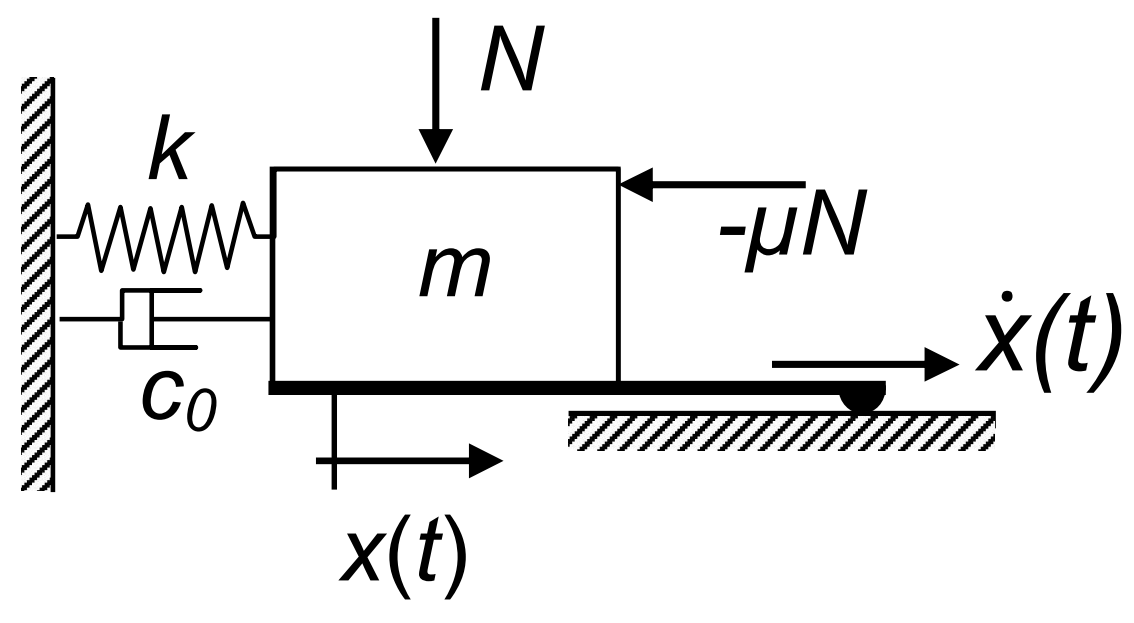


Fig. 2: Linear velocity-dependent friction coefficient

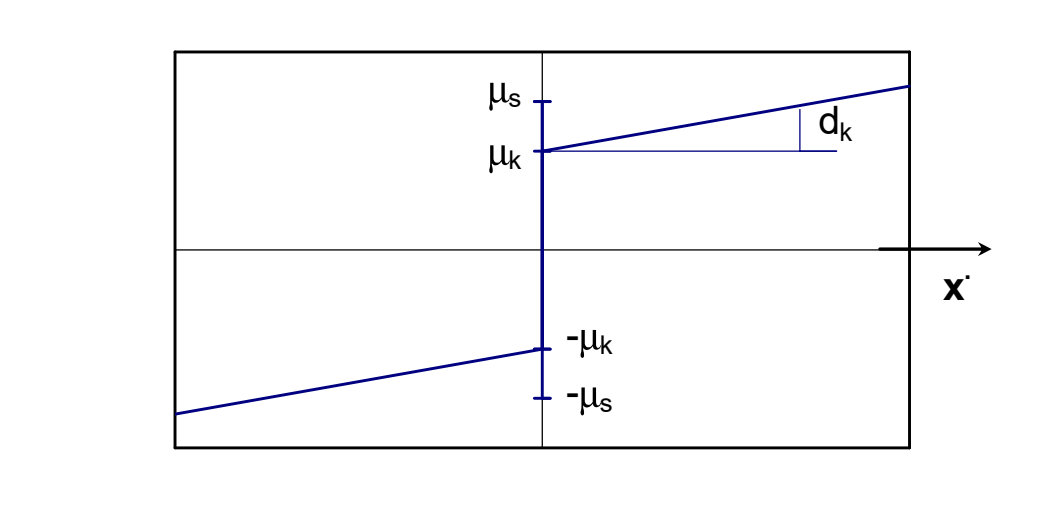

Fig. 2. Linear velocity-dependent friction coefficient

.

.
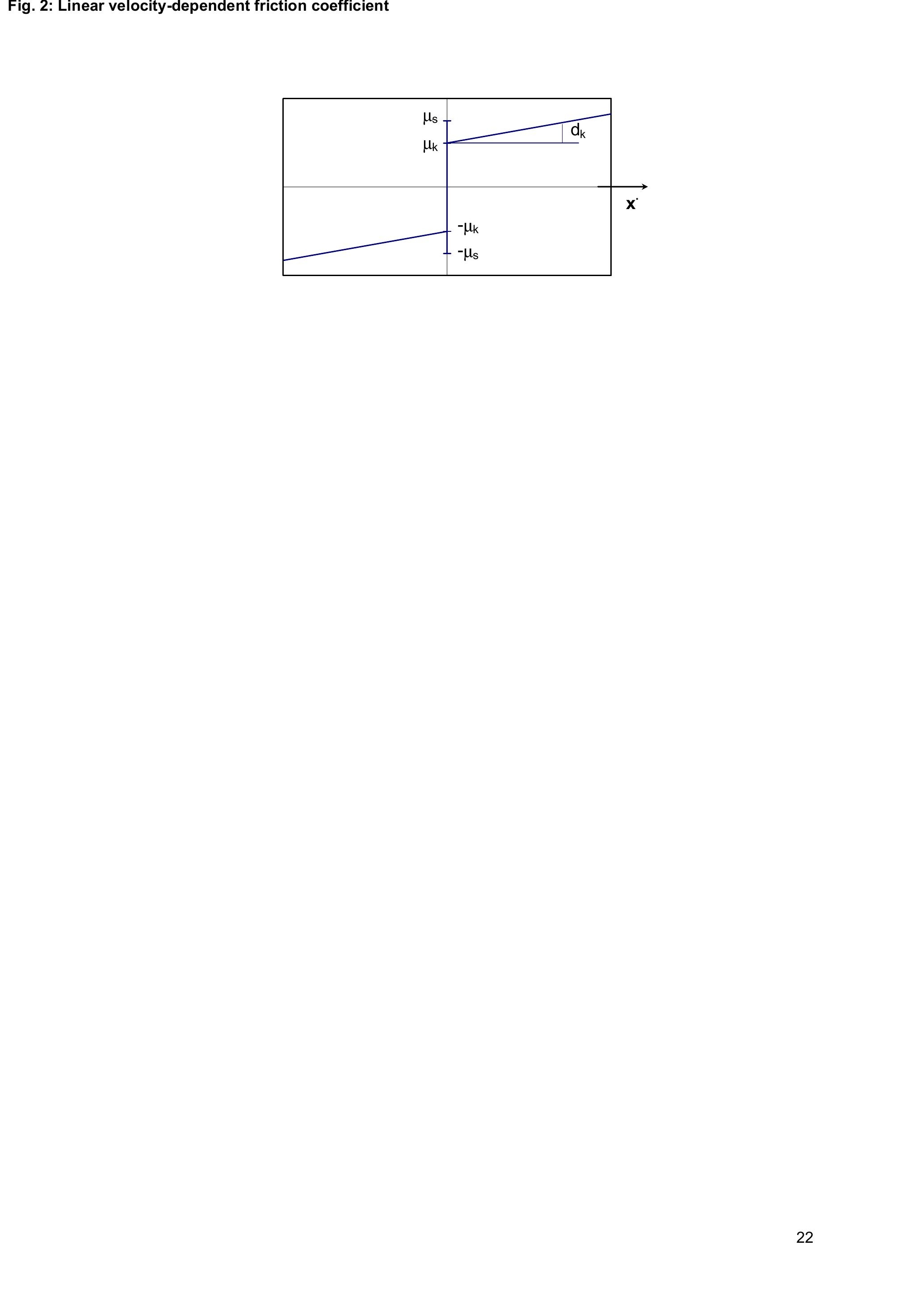
Fig. 3: Trajectory on the phase plane
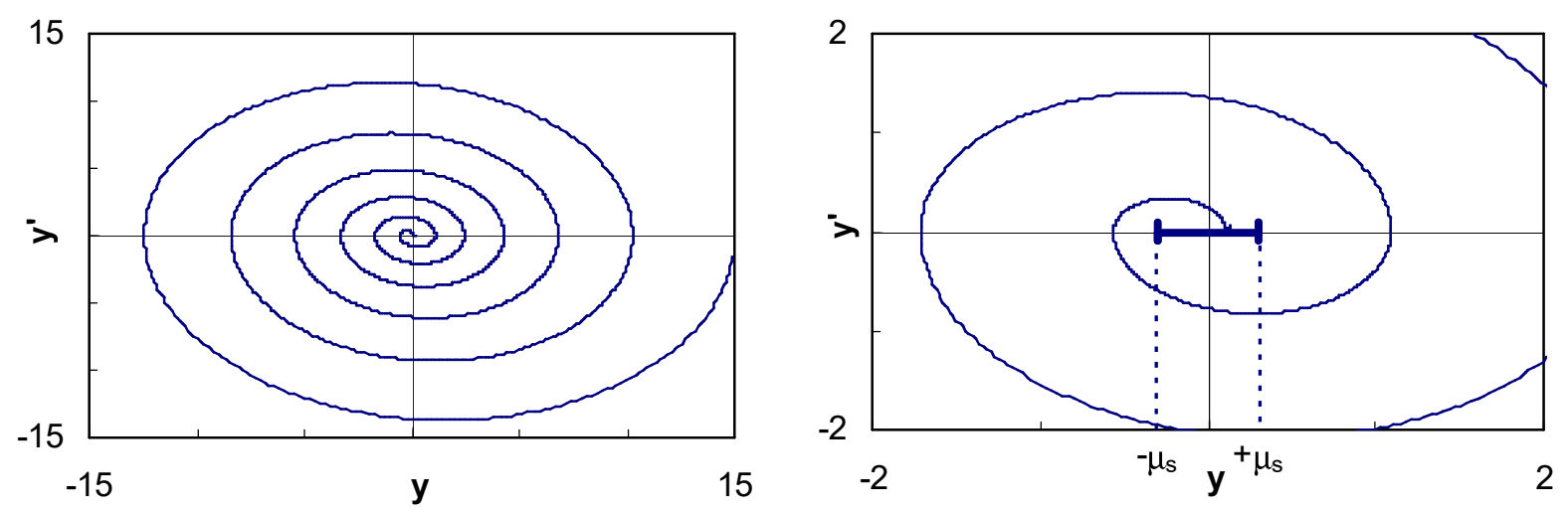
Fig. 4: Displacement (a) and velocity (b) time responses

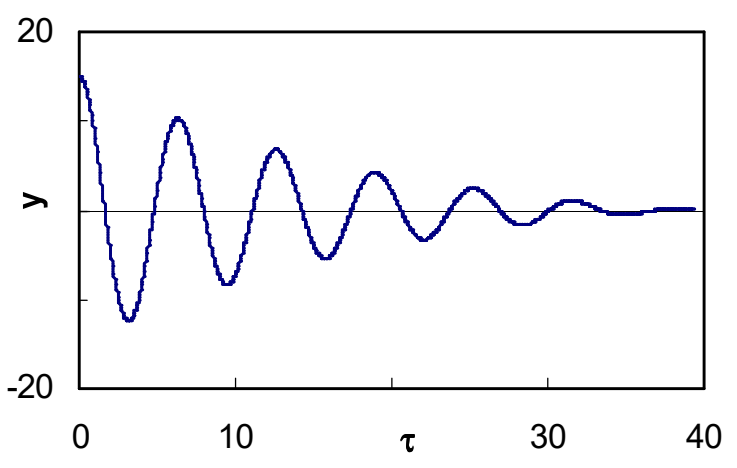

(a)

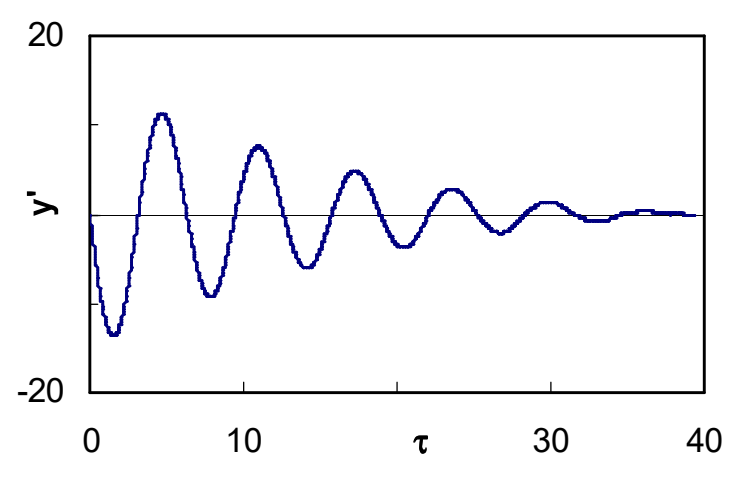

(b) 
Fig. 5: Energy decay curve (a) and averaged decay curve (b)

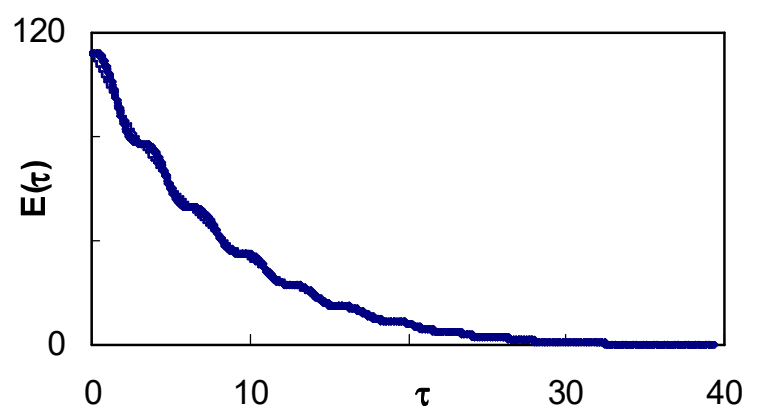

(a)

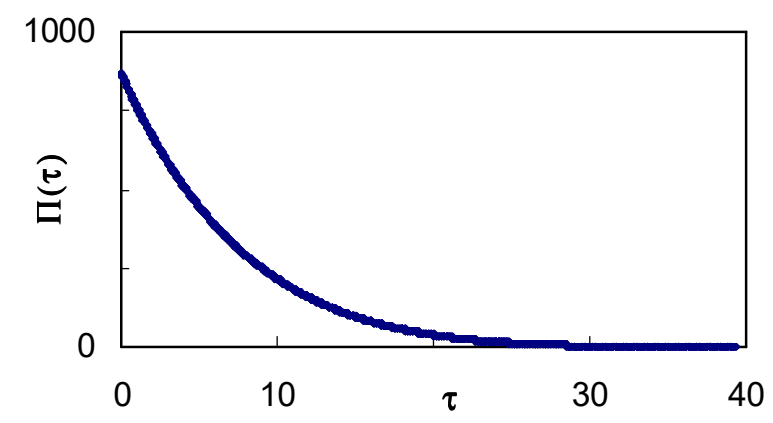

(b) 
Fig. 6: Schematic of the mechanical device

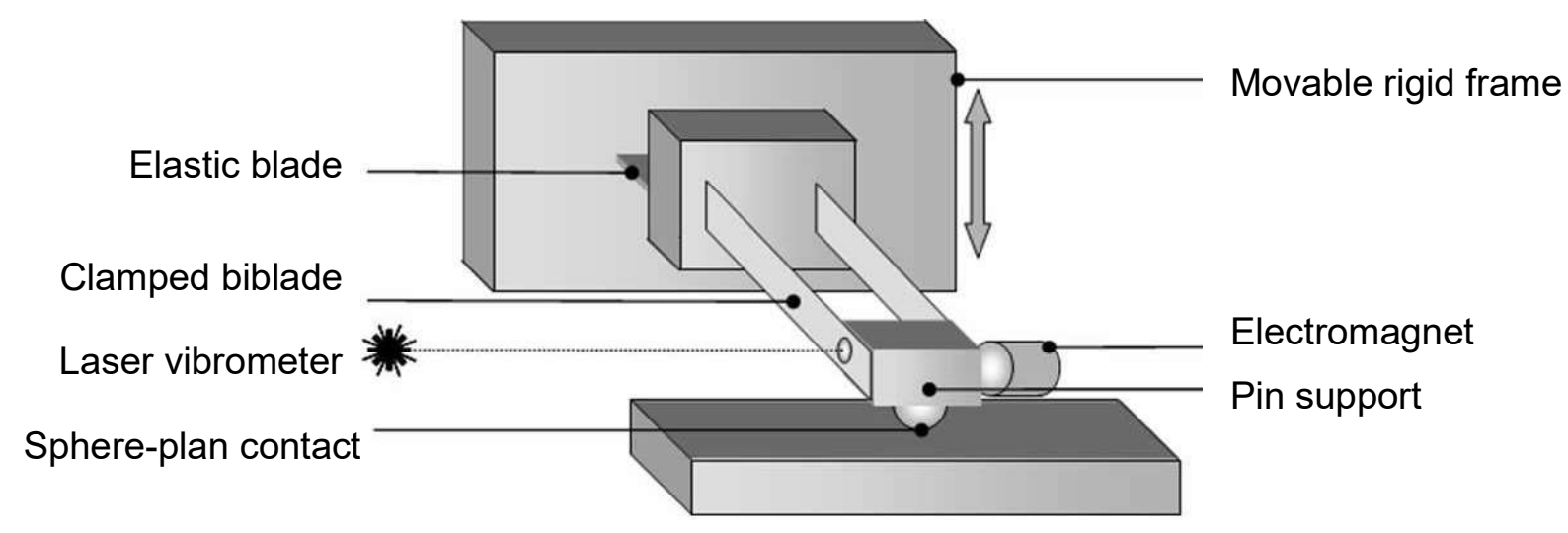


Fig. 7: Free response in the air without contact

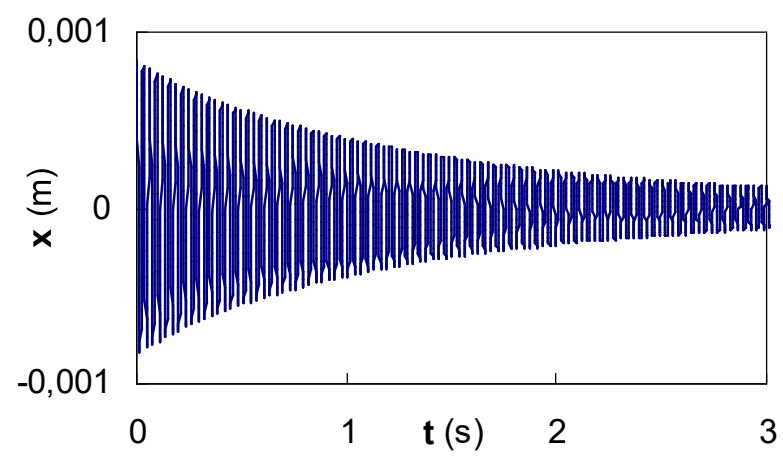

(a)

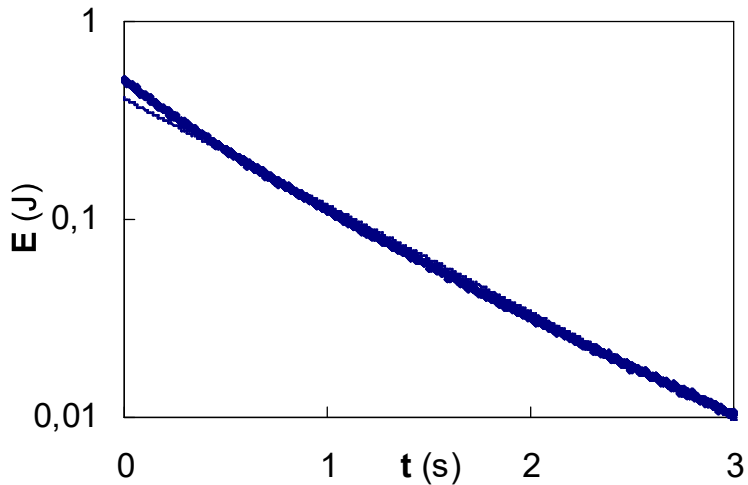

(c)

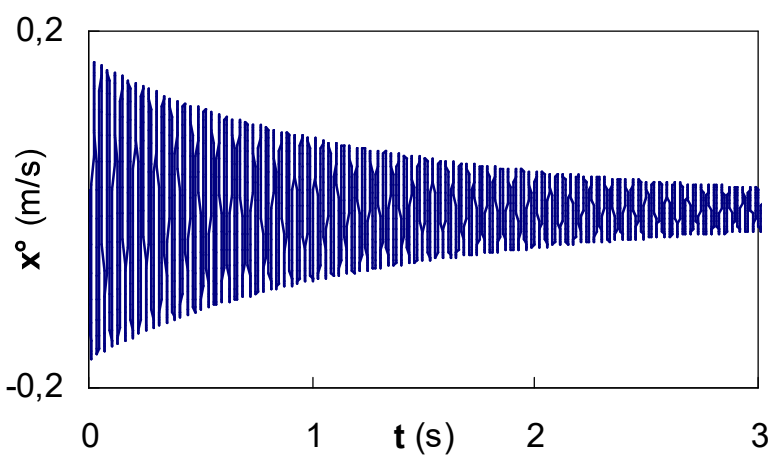

(b)

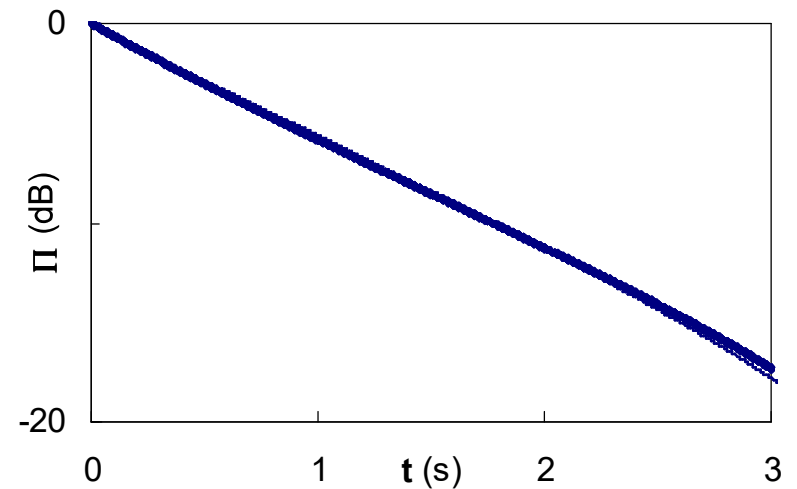

(d) 
Fig. 8: velocity responses (a) and energy decay curves (b)
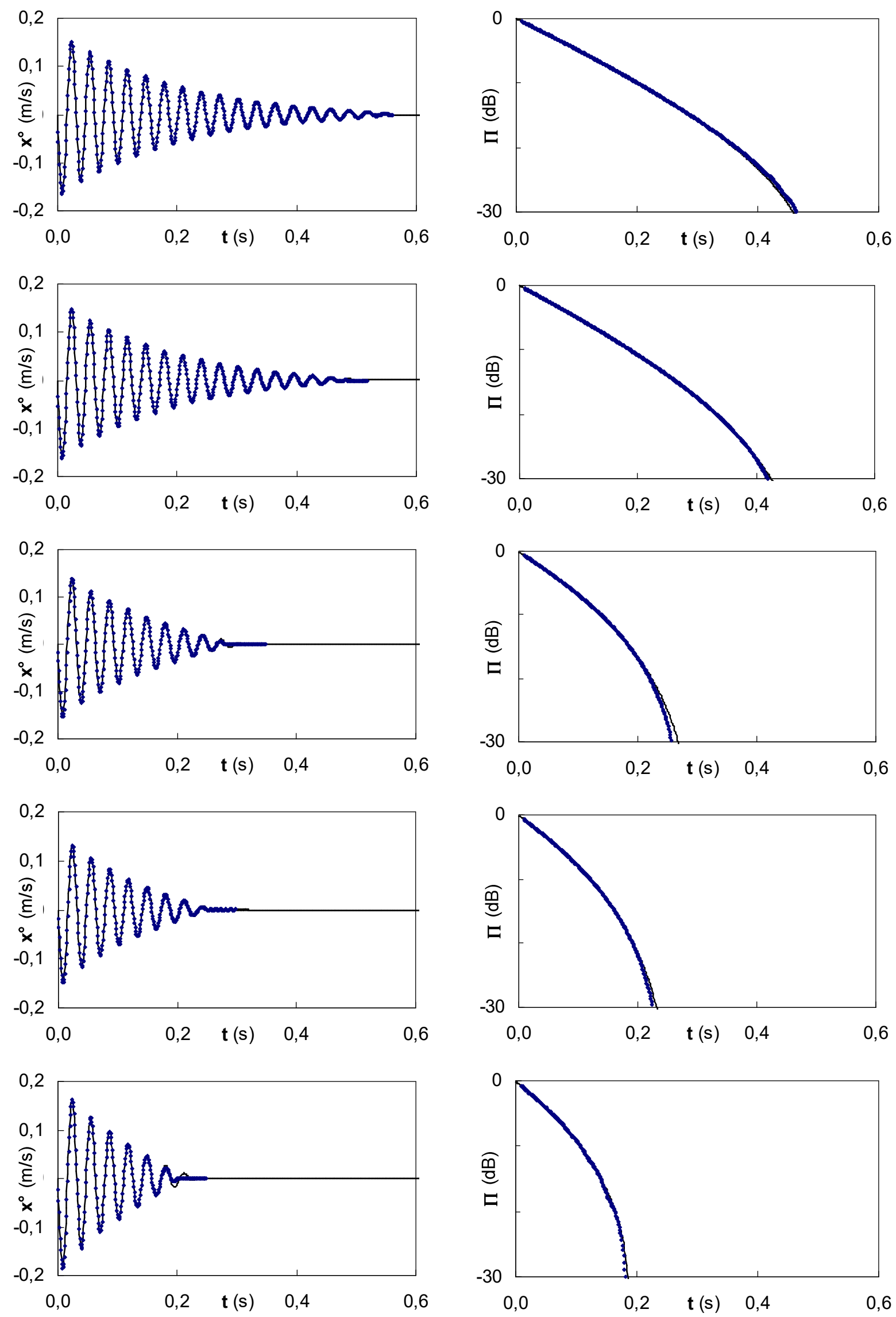

(a)

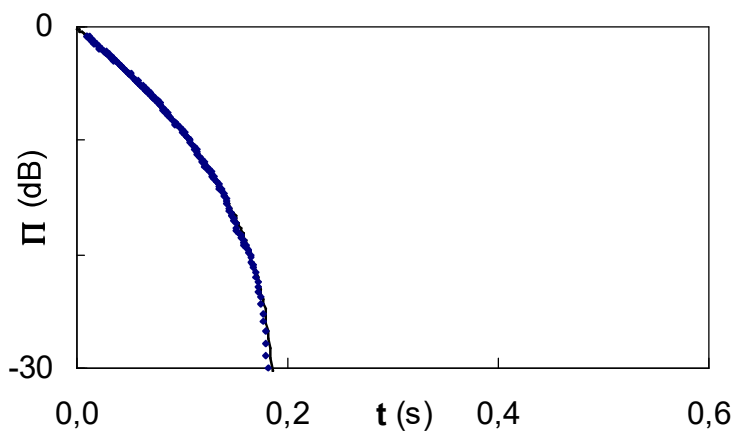

(b) 
Fig. 9: viscous damping (a) and friction (b) coeff vs load

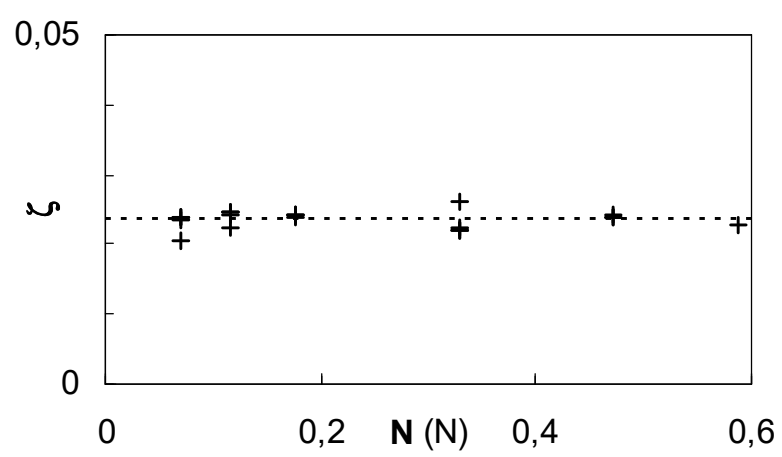

(a)

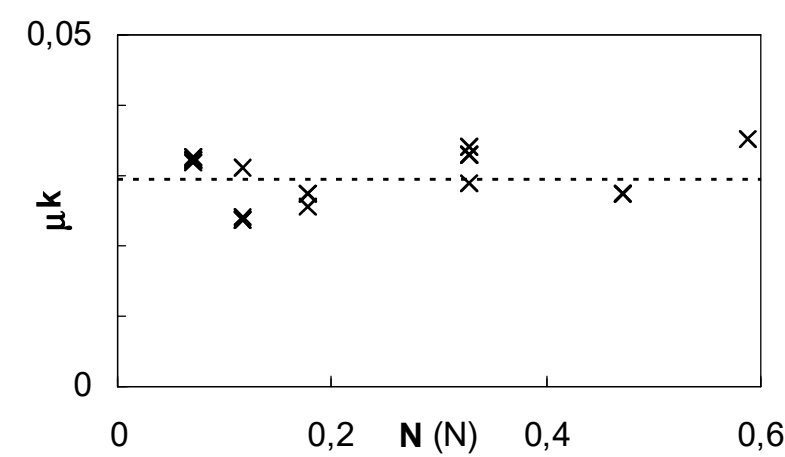

(b) 
Fig.10: viscous damping (a) and friction (b) coeff vs viscosity

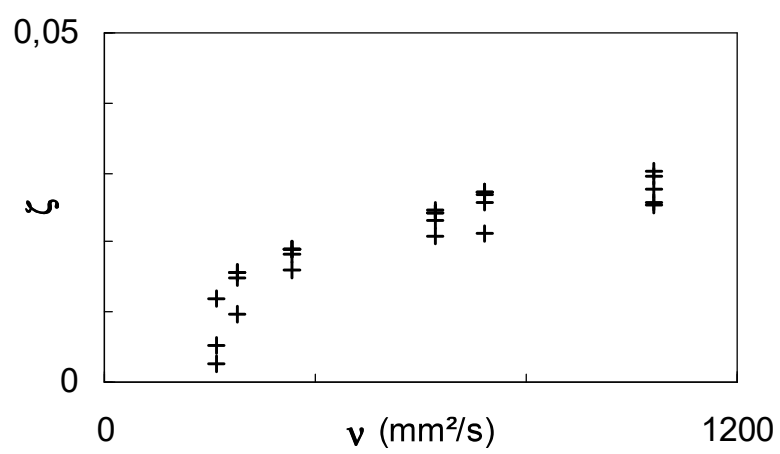

(a)

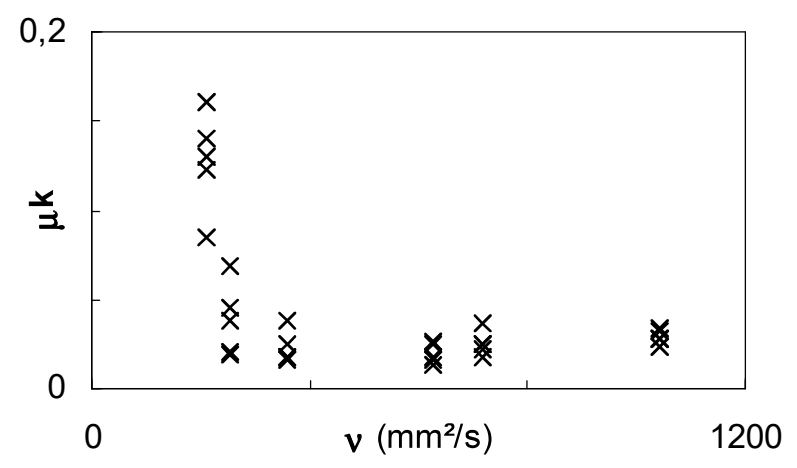

(b) 
Fig. 11: velocity response. Water mass fraction $6 \%(a), 9 \%(b)$

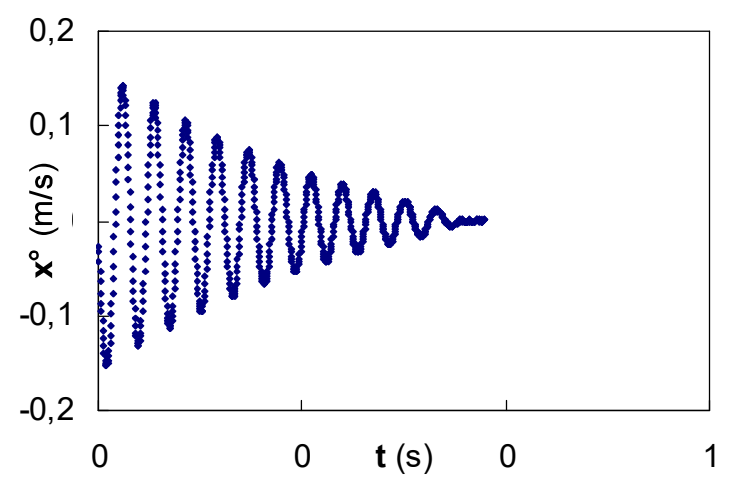

(a)

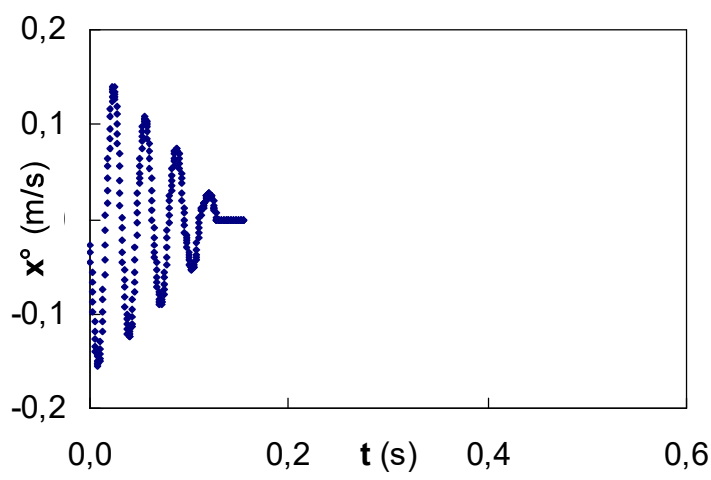

(b) 